\title{
Gamma Knife Stereotactic Radiosurgery (GKS) for the treatment of Vestibular Schwannomas (VS)
}

\author{
R. PERIN ${ }^{1}$, A. MOHAN ${ }^{2}$, H. MOISA ${ }^{3}$, A.V. CIUREA ${ }^{3}$ \\ ${ }^{1}$ Department of Neurosurgery, „Bagdasar-Arseni“ University Hospital, Bucharest, Romania \\ ${ }^{2}$ Department of Neurosurgery, Bihor County University Hospital, Oradea, Romania \\ ${ }^{3}$ Department of Neurosurgery, Sanador Medical Center Hospital, Bucharest, Romania; Department of \\ Neurosurgery, „Carol Davila“ University School of Medicine and Pharmacy, Bucharest, Romania
}

\begin{abstract}
Introduction. Vestibular schwannomas (VS) or acoustic neuromas (AN) (as they are known to neurosurgeons) are benign tumors which originate at the junction between central nervous system myelin and peripheral nervous system myelin - an area known as the Obersteiner-Redlich area. Acoustic neuromas represent a special pathologic entity for neurosurgeons due to their important symptoms which need to be adressed as soon as possible and due to the secondary disfunctions which may appear if the tumors are not treated appropriately.

Materials and methods. The study below presents the experience of the authors regarding the use of stereotactic radiosurgery for the treatment of acoustic neuromas. The authors focus on a population of 231 patients diagnosed and treated for VS over a period of 10 years at the "Bagdasar-Arseni" University Hospital in Bucharest. This study compares the impact of Gamma Knife Surgery when performed alone or in association with open microneurosurgery. The authors assessed patients with tumors ranging between 0.25 and $14 \mathrm{~cm}^{3}$ in volume. Gamma Knife Surgery is used successfully to treat VS, both independantly or in association with open microsurgery.

Results. The major complications of the treatment are represented by lack of response to treatment (less than $1 \%$ of patients) and cerebral edema (16.4\% of patients).

Conclusions. Given its multiple advantages, stereotactic radiosurgery is right now the tip of the spear regarding the therapeutical means available for VS. When the tumors met in daily practice are larger than the therapeutical resources of the devices used for Gamma Knife Surgery an open microsurgical approach may be attempted followed by stereotactic iradiation of the remnant tumor.

Given the large number of successes reported in the literature and the small number of complications but also the ever progressing technology used to improve Gamma Knife devices, the authors consider stereotactic radiosurgery to be a very good solution for the treatment of VS. Furthermore, the improvement potential this technique has grants it the potential to improve standard of treatment for acoustic neuromas and the quality of life in patients with this disease.
\end{abstract}

Keywords: Vestibular Schwannomas, acoustic neuromas, stereotactic radiosurgery, microneurosurgery, GKS, MRI 


\section{INTRODUCTION}

Vestibular Schwannomas (also known as acoustic neuromas) are tumors which were described for the first time by Sandifort in 1777 (1) and which were successfully removed for the first time by Sir Charles Balance. These benign tumors have a slow growth and are derived from Schwann cells in nerve roots (more specifically the Obersteiner-Redlich area in which tranzition is made from "central“ myelin - produced by oligodendrocytes - and "peripheral“ myelin produced by Schwann cells. These tumors appear frequently in the eight cranial nerve (VIII).

Vestibular Schwannomas (Fig. 1) are relatively rare tumors with a reported incidence between 0.5 and 1.7 cases for every 100,000 people. These tumors appear mostly in the 5th or 6 th decade of life and are rarely present in young patients (mostly in patients with neurofi-

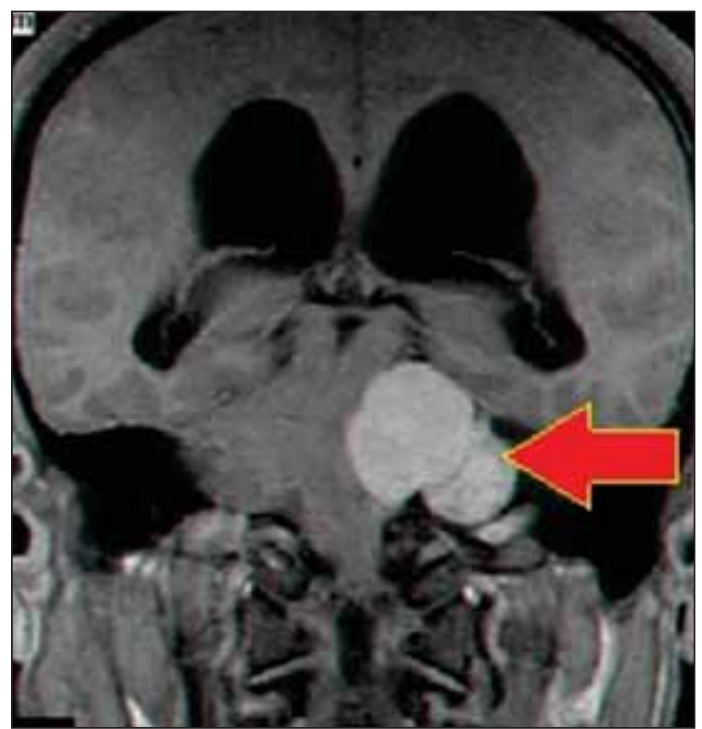

FIGURE 1. Giant acoustic neuroma with obstructive hydrocephalus. (MRI aspect)

Reproduced from Ciurea A.V. "Tratat de neurochirurgie" (in Romanian) Vol. I. 2010. bromatosis II). VS account for $6-8 \%$ of all intracranial tumors and between 25 and $33 \%$ of all the tumors in the posterior cranial fossa. $(2,6)$ Despite the lack of studies regarding the precise development of these tumors, a growing number of literature reports show that acoustic neuromas grow between 0.2 and $2 \mathrm{~mm}$ a year. (7)

Due to the astonishing work of Prof. Dr. Lars Leksell (a professor of neurosurgery from Sweden), Prof. Dr. Ladislau Steiner (a Romanianborn professor of neurosurgery) and Prof. Dr. Borje Larsson (a radiobiologist from Sweden), in 1967 at the Karolinska Institute of Sweden, the first Gamma Knife Stereotactic Radiosurgery unit is built, designed to deliver precise microdoses of radiation to various lesions of the human brain in order to destroy them. (8) Tipically, a Gamma Knife stereotactic radiosurgery machine has 201 sources of Cobalt-60 of about $30 \mathrm{Cu}$ rie. These sources are carefully enclosed into a shielded mechanism in such a way that no secondary beam of radiation is produced. Using a helmet with various orifices that is mounted on the head of the patient, the 201 beams of radiation converge into a single point, deep into the human brain where they induce sclerosis of the targeted lesion. (Fig. 2) This of course would not be possible without accurate MRI studies of the brain and the lesion.

If initially all VS had surgical indication as first intent solution, the progress of technology and the birth of Gamma Knife stereotactic radiosurgery changed the trend, making therapy more easy. Thus, today, Gamma Knife surgery is routinely used in countries in Western Europe while open microsurgery is reserved only as a back-up solution or is employed in very large tumors, where the structures of the cerebello-pontine angle and the posterior cranial fossa need to be decompressed.

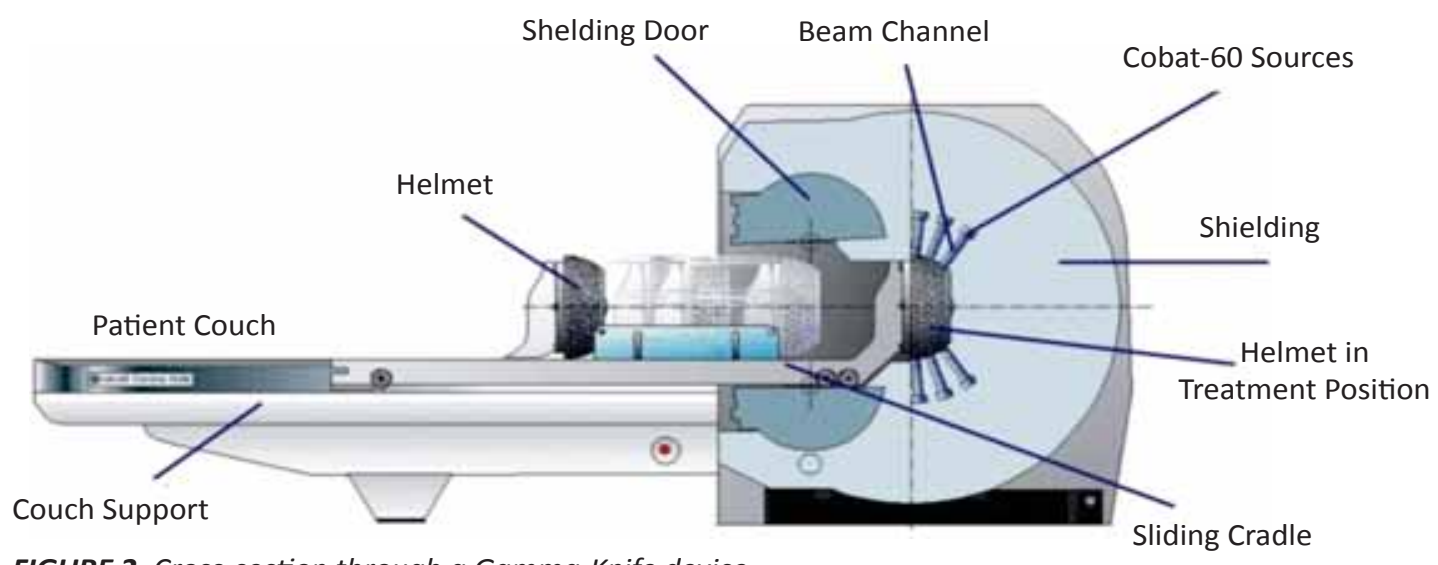

FIGURE 2. Cross-section through a Gamma-Knife device 
Stereotactic radiosurgery is a relatively new method which uses convergent beams of ionizing radiations to inactivate or destroy one or more well-defined targets in the Central Nervous System, without ever needing an incision. The main advantages of the method are the fact that the multiple beams of radiation have no impact on surrounding brain tissue, the ammount of time spent in the hospital is reduced to a minimum, there is no need for general anesthesia and the results of this technique are excellent while having minimal complications. Using this technique, control rates of up to 93$97 \%$ were achieved accompanied by hearing improvement in some cases. (10-15)

\section{MATERIALS AND METHODS}

The authors present a study of 231 consecutive patients, diagnosed, treated and followedup for VS over a period of 10 years (Jan. 2004 Jan. 2014) at the „Bagdasar-Arseni“ University Hospital in Bucharest. All the patients have, at some point during their treatment, received Gamma Knife Surgery, either as first intention treatment or as a follow-up treatment after open microsurgery. All the patients were sub- jected to a protocol consisting of Clinical Assessment, Computed Tomography imaging, Admission, MRI Imaging, GKS/Surgery + GKS, Recovery, Discharge, Follow-up. (See Table 1) The followup period ranges between 3 and 7 years and consisted of clinical and MRI investigations every 6 months for the first 3 years, after which MRI imaging was performed yearly. The authors excluded from the study all patients that had been admitted for tumor relapses.

During this study, the authors used a Model C Gamma Knife Surgery Device, outfitted with an advanced three-dimensional positioning system of the patient in relationship with the radiation sources. The System was installed at the „Bagdasar-Arseni“ University Hospital in 2004 and since then a number of more than 2194 interventions were performed. Out of the 2194 interventions, a number of 231 were for patients with VS. The mean tumor volume for the 231 patients was of $5.35 \mathrm{~cm}^{3}$, ranging from 0.25 $\mathrm{cm}^{3}$ and up to $14.0 \mathrm{~cm}^{3}$. For the treatment of these tumors the authors used between 1 and 15 doses of radiation with a marginal dose ranging between 12 and $14 \mathrm{~Gy}$, on isodoses ranging between 45 and $60 \%$. (See Table 1 for decision making algorhythm).

TABLE 1. Acoustic Neuroma decision making algorhythm Reproduced from „Radiosurgery Practice Guideline Initiative, Stereotactic Radiosurgery for patients with Vestibular Schwannomas. Radiosurgery Practice Guideline Report" \#4-06, 2006

\section{Management Algoritm for Acoustic Tumors}

Tumor Size,

Rainstem Compression

Intracanalicular Tumor

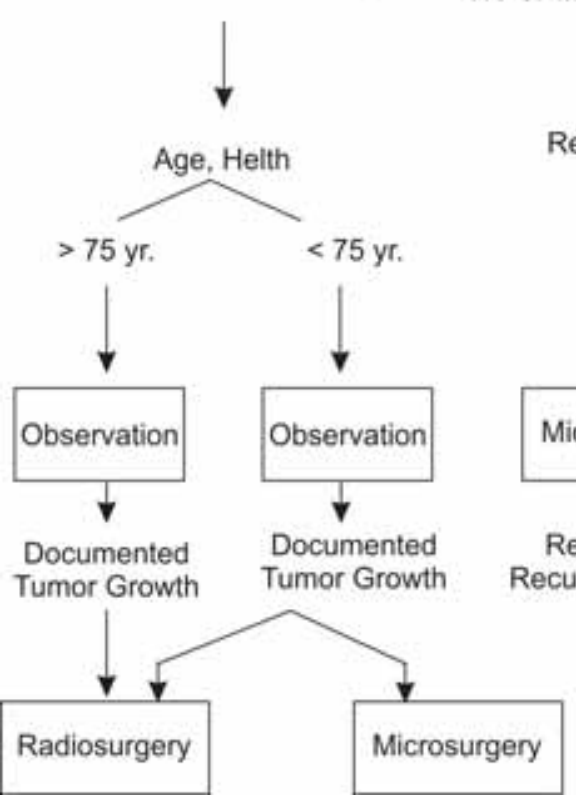

Tumor Diameter $<3 \mathrm{~cm}$, No or Mild Brainstem Compression

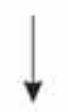

Review of Treatments, Goals, Patients' Choice

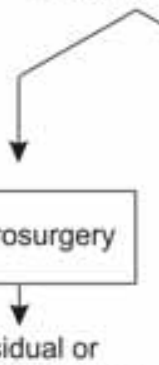

Residual or Recurrent Tumor
Tumor Diameter $>3 \mathrm{~cm}$, Symptomatic Brainstem Compression

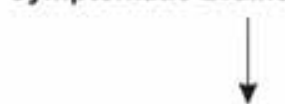

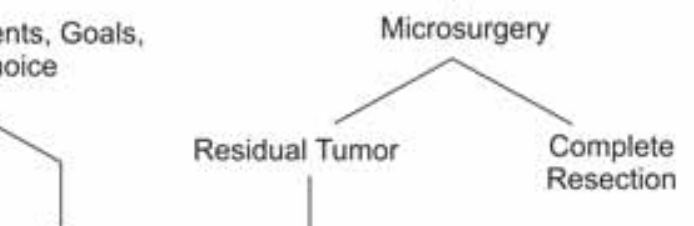

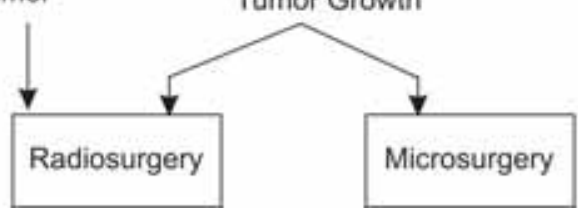


A number of 127 patients had been previously operated on, and were receiving secondary iradiation for the remnant tumor, while 104 patients received Gamma Knife Surgery as first intent therapy, given the fact that their tumors were smaller than $3 \mathrm{~cm}^{3}$ - which is an important criteria for successful first intent radiosurgery. Nevertheless, the critical volume of $3 \mathrm{~cm}^{3}$ can be achieved using an open surgical intervention followed by Gamma Knife Surgery - a technique used with great success in the case of large tumors in very difficult locations.

\section{RESULTS}

The results were divided into two groups based on whether the patient had received GKS as first intent therapy (Group 1) or whether the patients received GKS after prior microsurgery (Group 2).

Group 1: For the 104 patients that received Gamma Knife surgery, a number of 71 (68.2\%) patients showed tumor regression (Fig. $3 a$ and $3 b)$. A number of 31 patients $(29.8 \%$ ) did not show any sign of regression while the tumor maintained its dimension. A number of 2 patients $(2 \%)$ showed no response to treatment as the tumors grew (Fig. 4a and 4b).

Group 2: For the 127 patients that were operated on and then received Gamma Knife Surgery, 90 patients (71\%) showed shrinkage of the tumor, 33 patients $(26 \%)$ showed constant tumor dimension while 4 patients (3\%) showed a slow growth of the tumor.

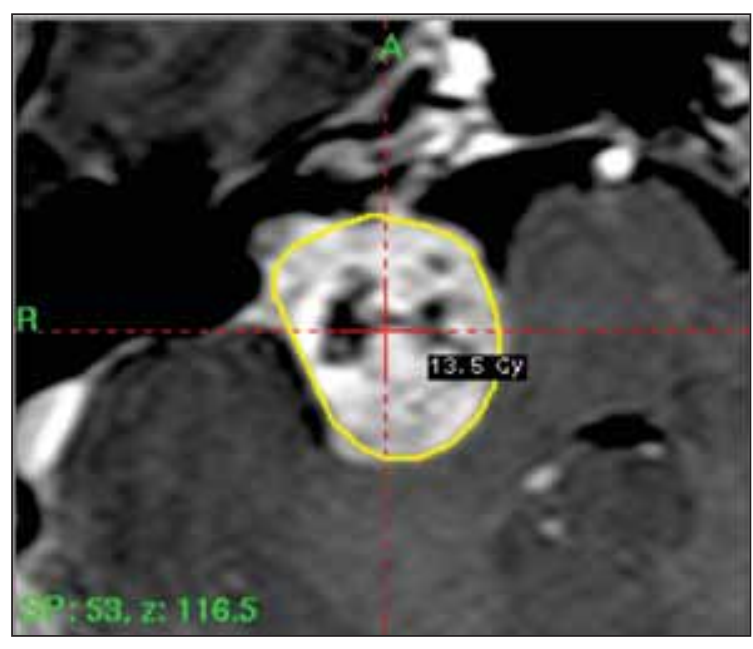

a

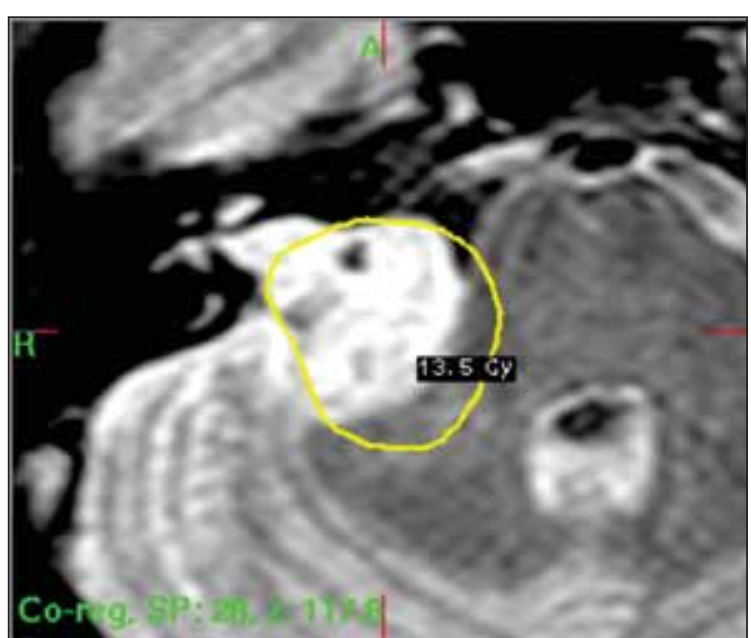

b

FIGURES $3 \boldsymbol{a}$ and $\mathbf{3 b}$. Vestibular Schwannoma during treatment (a) and check-up at two years; (b) Notice the decrease in size.
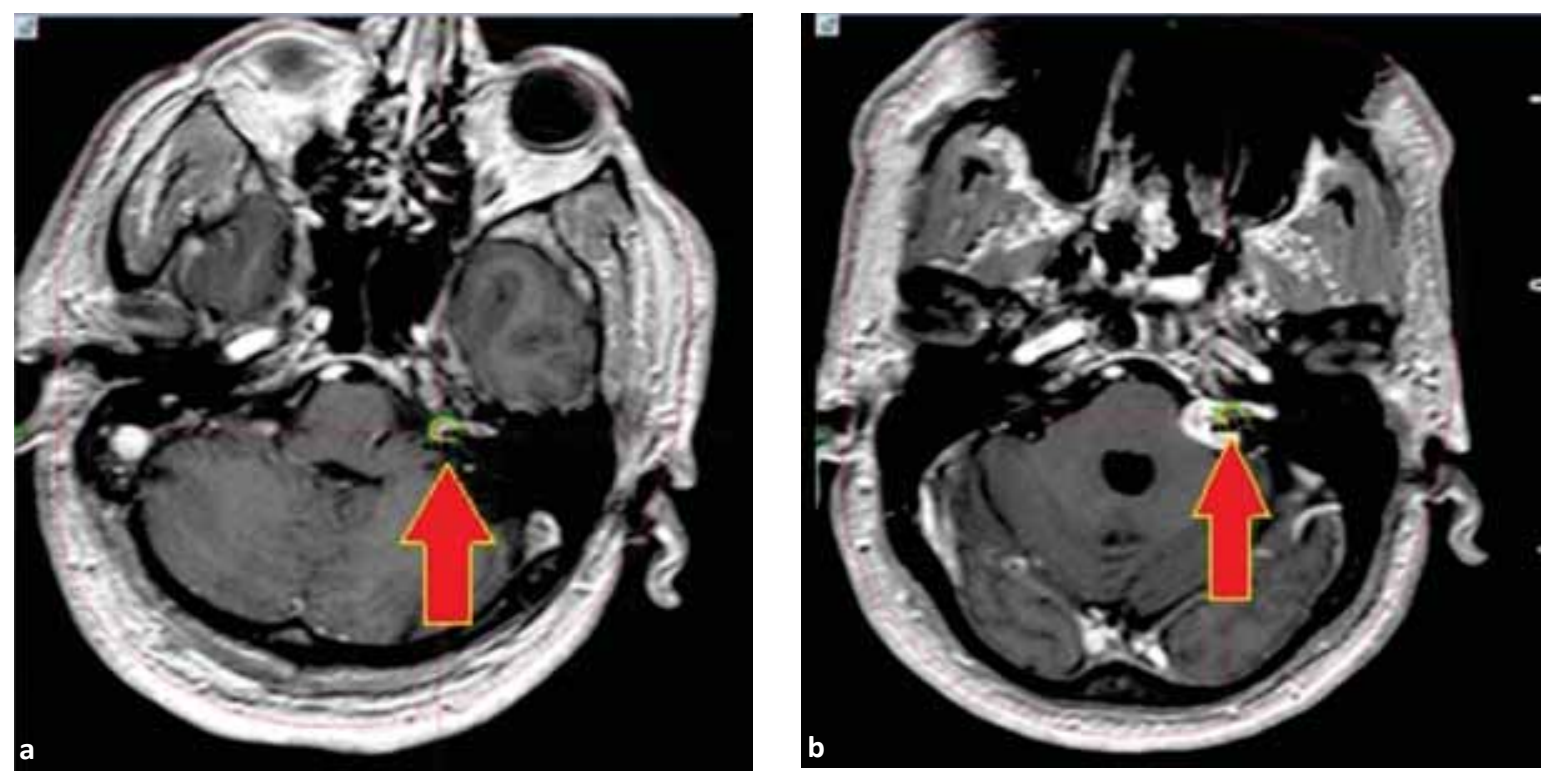

FIGURES $\mathbf{4} a$ and $\mathbf{4 b}$. Vestibular Schwannoma in a patient 4 years after Gamma Knife Surgery. Tumor size grew from $7 \mathrm{~mm}$ (a) to $15 \mathrm{~mm}$ (b) as the patient was unresponsive to GKS. The decision was taken to operate the patient. 


\section{COMPLICATIONS}

The follow up was performed using clinical and MRI studies performed at 6 months for a period of 3 years after which, the check-up was scheduled once a year. The first complication met in the 231 patients was the ineffectiveness of the treatment. A number of 2 patients $(0.85 \%)$ treated using GKS as first intention did not respond to the treatment and required open surgery to have the tumor removed. The second most important complication was postirradiation cerebral edema which was noticed in 38 cases $(16.4 \%)$ and treated appropriately.

Other frequent complications, which were met in patients that were operated on, were permanent and transient facial palsies noticed in 36 cases $(15.6 \%)$ of the patients, trigeminal palsies in 29 cases $(12.5 \%)$ with large tumors, and trigeminal neuralgia in 9 cases $(3.8 \%)$. Patients with large tumors $\left(>8 \mathrm{~cm}^{3}\right)$ showed good compliance with the treatment and had a very good evolution.

\section{CONCLUSIONS}

Gamma Knife Stereotactic Radiosurgery (GKS) for VS is one of the most advanced thera- peutical options availabe worldwide. When tumor size surpasses the therapeutical resources of the radiosurgery device, a microsurgical approach can be carried out to shrink the tumor and follow-up Gamma Knife Surgery is possible.

Given the large number of therapeutical successes reported and the small number of complications, the authors believe this method is highly valuable. As technique improves so does technology implemented in Gamma Knife machines. Three dimensional resolution increases, the radiation doses used get smaller and the patients'quality of life improves as literature quotes cases of hearing improvement. (14) Since the introduction of Gamma Knife Surgery, patients older than 75 with important comorbidities can benefit as well from treatment for vestibular schwannomas. $(15,16)$

The only reasonable doubt regarding Gamma Knife Surgery today is the possible malignant degeneration of acoustic neuromas, however, up until this moment there is no mention in the literature of such a case. $(2,9)$

The main and most useful tool for follow up is high resolution MRI scanning as it allows the surgeon to notice the growth pattern of the tumor and greatly influences the therapeutical protocol.

\section{BIBLIOGRAFIE}

1. Cushing H. Tumors of the Nervus Acusticus and the Syndrome of the Cerebellopontine Angle. Philadelphia: Saunders, 1917

2. Misra B.K., Management of Vestibular Schwannomas: in Kalangu K., Kato $Y$. Essential Practice of Neurosurgery. Access Publishing, Nagoya, pag. 322-326, 2011.

3. Briggs R.J., Fabinyi G., Kaye A.H. Current management of acoustic neuromas: review of surgical approaches and outcomes. J Clin Neurosc. 7:521-526, 2000.

4. DeMonte F., Al-Mefty O. Neoplasms and the cranial nerves of the posterior fossa. In: Barrow DL. Surgery of the cranial nerves of the posterior fossa. Park Ridge, IL, pag. 234-253, 1993

5. Sampath P., Rini D., Long DM. Microanatomical variations in th cerebellopontine angle associated wih vestibular schwannomas: A retrospective study of 1006 consecutive cases. J Neurosurg 92:70-78, 2000
6. Grossman R.G., Loftus C.M. (eds.). Principles of Neurosurgery, ed 2. Philadelphia: Lippincott-Raven, 1999

7. Samii M., Matthies C. Management of 1000 vestibular schwannomas: surgical management and results with an emphasis on complications and how to avoid them. Neurosurgery 40:11-21, 1997

8. Nica D.A., Mohan D., Moisa H.A., Ciurea A.V. The legacy of Ladislau Steiner (1920-2013): The neurosurgeon who won the heart of the World. Neurosurg Rev 37: 175-178(2014)

9. Rowe J., Grainger A., Walton L., Silcocks P. Risk of malignancy after Gamma Knife Stereotactic Radiosurgery. Neurosurgery 60:60-66, 2007.

10. Prasad D., Steiner M., Steiner L. Gamma Knife Surgery for vestibular schwannoma. J. Neurosurg, 92:745-750, 2000;

11. Flickinger JC., Kondziolka D., Niranjan

A., Lunsford LD. Results of acoustic neuroma radiosurgery: An analysis of 5 years experienceusing current methods. J. Neurosurg 94:1-6; 2001;

12. Lunsford L.D., Niranjan A., Flickinger J.C. et al. Radiosurgery of vestibular schwannoma: Summary of experience in 829 cases. JNS: 102 (Suppl.) 195-199; 2005

13. Kano H., Kondziolka D., Khan A., Flickinger JC., Lunsford LD. Predictors of hearing preservation after stereotactic radiosurgery acoustic neuroma. J. Neurosurg 2009: Mar 13

14. Niranjan A., Lunsford LD., Flickinger JC et al. Can hearing improve after acoustic tumor radiosurgery? Neurosurg Clin N Am: 10; 305-316;1999:

15. Regis J., Pellet W., Delsanti C et al. Functional outcome after Gamma Knife Surgery or Microsurgery for Vestibular Schwannomas. J Neurosurg 97: 10911100; 2002 